\title{
The stratigraphical organisation of the microvascular systems of the porcine vocal folds
}

\author{
H. Reinhard, A. Lang, H. Gasse \\ Institute of Anatomy, University of Veterinary Medicine Hannover, Germany
}

[Received 27 February 2014; Accepted 14 April 2014]

The cranial and caudal vocal folds (CraF, CauF) of the glottis of adult minipigs (11-27 months; $n=12$ ) were examined after immunohistochemical application of polyclonal anti-von-Willebrand-Factor and anti-Smooth-Muscle-Actin in serial paraffin sections. This examination aimed at a stratigraphical analysis of microvessels; data were compared with findings in humans which had been reported in the literature.

(1) The distribution of the microvessels was very heterogeneous in the CraF and in the CauF, but a common pattern existed in both. (2) Characteristic vascular zones and rows were detected; each of them displayed a specific distribution and density of blood capillaries, arterioles, venules, lymphatic capillaries, and lymphatic precollectors. (3) A striking feature was the presence of a subepithelial Avascular Band and of a focal Avascular Area within the lamina propria of the fold's crests. (4) The vascular zones, the rows, the Avascular Band, and the Avascular Area could be allocated to specific layers of the lamina propria: subepithelial, superficial, intermediate, deep layer. (5) The loose Avascular Area at the level of the superficial layer of the lamina propria (in both CraF and CauF) corresponded to Reinke's space in humans in terms of structure and location. (6) The direction/ /course of blood and lymphatic microvessels shared common features with that of the human vocal fold. (Folia Morphol 2014; 73, 4: 439-448)

Key words: anti-von-Willebrand-Factor, anti-Smooth-Muscle-Actin, lymphatics, paraffin sections

\section{INTRODUCTION}

The viscoelastic properties of the vocal folds have a major impact on the vibratory movement during phonation [9]. These properties are related to several features: Firstly, they depend on the amount and distribution of collagenous and elastic fibres, which characterise the stratigraphical organisation of the lamina propria [3, 11,49]; secondly, they are influenced by the local distribution of the amorphous Ground Substance [26].

The interstitial fluid in the Ground Substance and, in particular, the molecules in it affect and often con- trol the viscosity of the vocal fold's tissue [6]. In terms of this, the mechanisms of inflow, binding (stasis), and outflow of fluids deserve major attention. In this paper, stress shall be put on the structural component of these systems, i.e. blood capillaries and lymphatic capillaries/lymphatic precollectors. The description of their local distribution shall complement the detailed stratigraphical examinations of the minipig's vocal folds [17], whose lamina propria is subdivided into 4 layers: subepithelial, superficial, intermediate, and deep layer. These layers are characterised by different widths, fibre qualities, and fibre densities.

Address for correspondence: Dr H. Gasse, Institute of Anatomy, University of Veterinary Medicine Hannover, Bischofsholer Damm 15, 30173 Hannover, Germany, tel: 0511 8567573, fax: 0511 856827214, e-mail: Hagen.Gasse@tiho-hannover.de 
In particular, band-like or focal regions, which were conspicuous because of their sparse number of fibres [17] and blood vessels, attracted special attention, as they may be related to Reinke's space in the human glottis. In humans, Reinke's space may become frequently affected by pathological conditions, especially by Reinke's oedema [15, 32, 33, 35], which influences the voice [8].

In the human vocal fold, the lymph vascular system $[16,34,18-20,44-47]$ and the blood vascular system $[23,25,34]$ have been studied intensively. However, topographical and stratigraphical relations have not been fully considered. Consequently, the location of the different microvascular networks in situ and their attribution to certain histological layers of the lamina propria still have to be evaluated. This is even more relevant because of the species-specific anatomical characteristic of the pig's glottis. A distinction between its phoniatrically relevant cranial and caudal fold (for details see [17]) is mandatory, especially if the minipig is intended to be used as a model for humans.

A lightmicroscopical approach on tissue sections from the minipig was used in order to evaluate the topographical distribution simultaneously of both, blood and lymph vascular structures in situ. This was performed by the immunohistochemical application of polyclonal anti-von-Willebrand-Factor (anti-SMA) and anti-Smooth-Muscle-Actin (anti-vWF).

\section{MATERIALS AND METHODS}

The specimens were taken from the larynges of female minipigs (aged 11-27 months, $n=12$ ) of the Göttinger Minipig and Mini Lewe Minipig breeds. All animals were obtained and euthanased primarily for the anatomical dissection classes at the University of Veterinary Medicine Hannover. All related procedures were performed in accordance with the Guidelines of the European Convention for the Protection of Vertebrate Animals used for Experimental and other Scientific Purposes (86/609/EEC). Respecting this, an approval by the Lower Saxony State Office for Consumer Protection and Food Safety was not necessary. The animals were euthanased either by injection (by a veterinarian) of $1.5 \mathrm{~mL} / 10 \mathrm{~kg}$ Euthadorm ${ }^{\circledR}$ (pentobarbital sodium) or by captive bolt stunning and consecutive bleeding; they showed no signs of larynx-associated diseases upon examination.

\section{Histological procedures}

The larynges were excised immediately after euthanasia and immersion-fixed in Bouin's solution

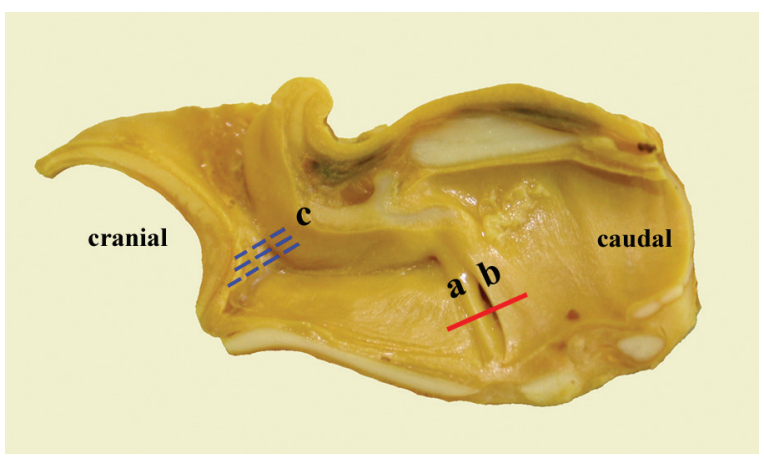

Figure 1. Bisected porcine larynx; $a$ - cranial fold; $b$ - caudal fold; the opening of the laryngeal ventricle lying in-between; $\mathrm{c}$ - position of the vestibular ligament.

(saturated aqueous picric acid, filtered acid-free formaldehyde $37 \%$, acetic acid $98 \% ; 25: 10: 1$ ) at room temperature for $48 \mathrm{~h}$. For histological processing, the larynx was bisected in the median plane. Then, the glottis was excised from each half and cut transversely at its midportion (Fig. 1). These specimens were then treated with the standard procedure of alcoholic dehydration and paraffin embedding. Serial cross sections $(5-8 \mu \mathrm{m})$ were alternately stained either with Masson's Trichrome or haematoxylin and eosin. Four other sections were immunostained with polyclonal anti-vWF. Another 4 sections were immunostained with monoclonal anti-SMA Clone 1A4. One section of each immunostain was selected for the histomorphometrical examination. All sections represented the midportion of the folds.

\section{Immunohistochemical staining: anti-vWF, anti-SMA}

The paraffin cross sections were deparaffinised in xylol and rehydrated in a graded alcohol series. Endogenous peroxidase was inhibited using 0.6\% hydrogen peroxide in alcohol $80 \%$ for $30 \mathrm{~min}$. Three washes ( $5 \mathrm{~min}$ each) were performed with $0.05 \mathrm{M}$ trisphosphate-buffered saline with Tween TBS-T pH 7.4 between each step of the procedure, if not otherwise stated. Antigen retrieval was performed differently: the sections designed to be immunostained with anti-vWF were pretreated with proteinase $\mathrm{K}$ (Sigma-Aldrich) in phosphate-buffered saline 1:200 at $37^{\circ} \mathrm{C}$ for $25 \mathrm{~min}$; the sections designed to be immunostained with anti-SMA were placed in a water bath containing citric buffer (pH 6.0) and heated at $95-99^{\circ} \mathrm{C}$ for $20 \mathrm{~min}$. In order to prevent non-specific binding of antibodies, all sections were treated with normal goat serum at room temperature for $20 \mathrm{~min}$. 

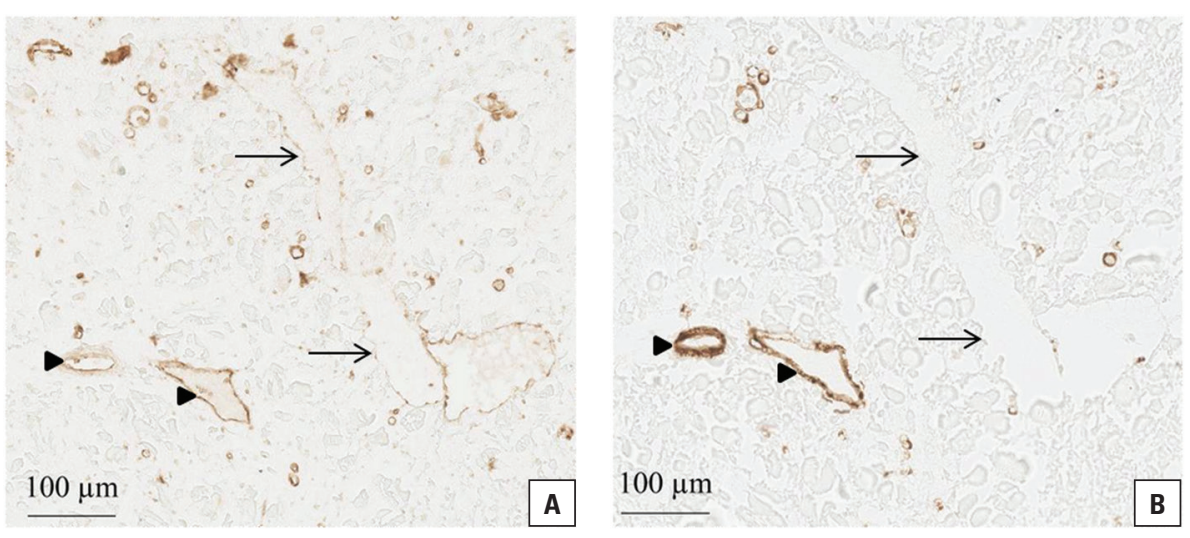

Figure 2. Immunohistochemical staining of two adjacent serial paraffin cross sections of the porcine glottis; $\mathbf{A}$. Anti-von-Willebrand-Factor detected endothelium of blood vessels (arrowheads) and endothelium of initial lymphatics (arrows); B. Anti-Smooth-Muscle-Actin (anti-SMA) detected blood vessels (arrowheads), but no initial lymphatics (arrows: anti-SMA negative lymph capillary).

Table 1. Immunoreactivity of anti-von-Willebrand-Factor (anti-vWF) and anti-Smooth-Muscle-Actin (anti-SMA) in vascular cells, as recorded in the literature

\begin{tabular}{lcccc}
\hline & Blood vascular endothelium & Lymph vascular endothelium & Smooth muscle cells & Pericytes \\
\hline Anti-vWF $[13,28,30]$ & + & + & - & - \\
Anti-SMA $[27,28,38,39]$ & - & - & + & + \\
\hline
\end{tabular}

Primary antibodies were applied for $16-18 \mathrm{~h}$ at $4-8^{\circ} \mathrm{C}$ at a dilution of 1:400 (anti-vWF; Dako, Hamburg, Germany) or at a dilution of 1:100 (anti-SMA Clone 1A4; Dako, Hamburg, Germany). The sections were then incubated with Dako EnVision + System/HRP (a dextran polymer with secondary antibody and horseradish peroxidase; Dako, Hamburg, Germany) for $30 \mathrm{~min}$. The reaction was visualised by exposure to diaminobenzidine in a substrate buffer (Dako, Hamburg, Germany). Afterwards, the sections were washed 3 times $(5 \mathrm{~min}$ ) with phosphate buffered saline PBS pH 7.2, and with running tap water for $10 \mathrm{~min}$. Appropriate negative controls by omitting the antibody were run concurrently for each used antibody.

\section{Distinction between blood vessels and initial lymphatics: anti-vWF, anti-SMA}

Different types of blood vessels, and initial lymphatics, were distinguished by comparing adjacent serial sections which had been exposed to either anti-vWF or anti-SMA; the procedure of distinction was based on the expression of VWF and smooth muscular alpha-actin in different cells (Fig. 2), as reported in the literature (Table 1).

\section{Histomorphometric examination of the microvessels}

The stained cross sections were digitised ('Scan Scope' Aperio) and subsequently histomorphometrically examined with the aid of the graphics editing program Adobe Photoshop CS 3 Extended 10.0.1 (Adobe Systems). In a preliminary study [31], a common pattern of vascular distribution had been found for both the cranial and caudal vocal folds (CraF, CauF). This pattern was used to make a stratigraphical subdivision of the examined tissue areas. The key features of this common pattern, as shown in Figure 3, were: A subepithelial Zone $A$, subdivided in Row 1 and Row 2; a subsequent Zone $B$; and an additional Zone $\mathrm{C}$ (in the CraF only).

Furthermore, the CraF and the CauF were separated into 4 regions: cranial side, caudal side, crest, and ventricular fundus (Fig. 3). This separation was achieved as follows: The CraF and the CauF were defined as the parts which extended from the laryngeal wall into the lumen. An imaginary 'orientation line' was drawn from the cranial surface of the CraF's base to the caudal surface of the CauF's base (red, dashed line in Fig. 3); the ventricular fundus was located underneath this line. Then another line (i.e. blue lines in Fig. 3) was placed half way between the orientation line 


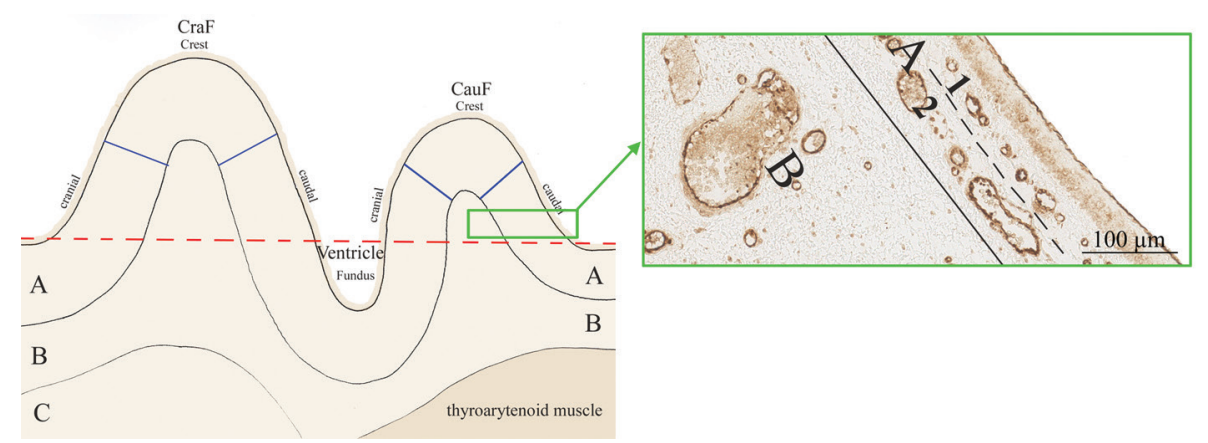

Figure 3. Graphical representation of a cross section of a porcine glottis with its cranial fold, caudal fold, and ventricle (CraF, CauF). The regional separation (cranial side, crest, caudal side, fundus) and the stratigraphical subdivision (Zone A, B, C) are shown. Inset: histological representation of the caudal part of the CauF with marked Zone A, subdivided into Row 1 and Row 2, and Zone B. Paraffin section, anti-von-Willebrand-Factor.

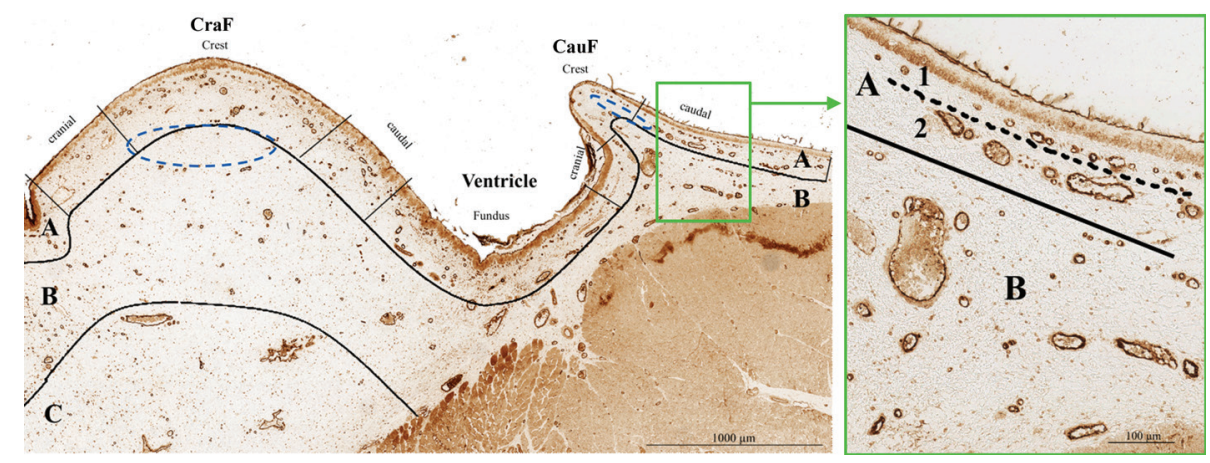

Figure 4. Cross section of a porcine glottis with its cranial fold (CraF), caudal fold (CauF), and ventricle. The common distribution pattern (Zones A, B, C) and the distinct Avascular Areas (blue dashed lines) are illustrated. Inset: magnified image of the caudal part of the CauF with marked Zone A, subdivided into Row 1 and Row 2, and Zone B. Paraffin section, anti-von-Willebrand-Factor.

and the highest point of the fold; this line separated the 'crest' from the 'cranial' and 'caudal' side of each fold. The placement of these division lines had to be specially adapted for each glottis of the different animals because of the large variability in the fold's shape and size; (e.g., the CauF often appeared bent in the cranial direction).

The type of blood vessels was distinguished on the basis of their calibres, i.e. blood vessels smaller than $10 \mu \mathrm{m}$ were designated as blood capillaries, while blood vessels larger than $10 \mu \mathrm{m}$ were designated as arterioles, and venules. The lymphatic vessels - i.e. lymphatic capillaries and precollectors - were referred to as 'initial lymphatics'.

In every zone and row of the 4 regions, 10 randomly selected distances between the profiles of blood capillaries, arterioles and venules, and initial lymphatics were measured. If fewer than 11 vascular profiles were encountered in a region, these were all included in the measurements. The mean values of every minipig were calculated and listed - for every zone, and every row of the different regions. From these individual mean values, a common mean value of all minipigs of the same age group was calculated - for every zone, and every row of the different regions. These mean values were taken as a descriptive equivalent of the vascular density within the different regions of the folds.

\section{RESULTS}

\section{Common distribution pattern}

The distribution of the microvessels was very heterogeneous in the CraF and in CauF, but basically, a common pattern existed in both (Fig. 4): A highly vascularised, 100-300 $\mu \mathrm{m}$ wide 'Zone A' was located near the epithelium. This zone comprised vascular profiles arranged in two rows: 'Row 1' consisted of blood ca- 


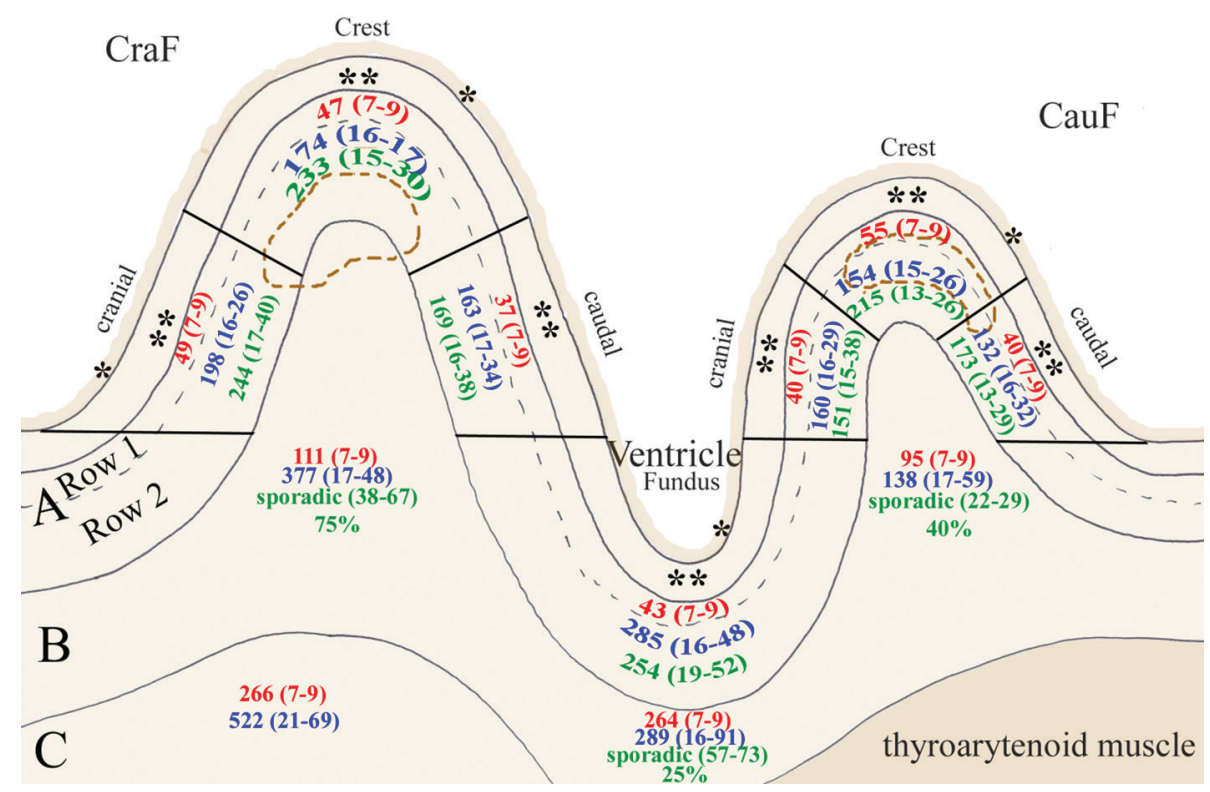

Figure 5. Graphical representation of a cross section of a porcine glottis with its cranial fold (CraF), caudal fold (CauF), and ventricle, to display distances between vascular profiles, and vascular diameters. The folds and the ventricle are covered by the epithelium $\left({ }^{*}\right)$; there is a distinct Avascular Band ${ }^{* *}$ ) underneath. The distances between the vascular profiles (coloured numbers without parentheses) and the diameters of the vascular profiles (coloured number in parentheses) are displayed. The colour code: blood capillaries (red), arterioles and venules (blue), lymphatics (green). Note the distinct Avascular Area (brown, dashed lines) in CraF and CauF. Note also that in Zone B the lymphatics occurred only sporadically; the percentage (i.e. $75 \%, 25 \%, 40 \%$ ) indicates in how many of the minipigs lymphatics were found.

pillaries, and 'Row 2' consisted of arterioles and venules (see inset in Fig. 4). Lymph capillaries and precollectors were almost exclusively distributed within Row 2.

'Zone $\mathrm{B}^{\prime}$ - approx. $1200 \mu \mathrm{m}$ wide in the CraF, 200-550 $\mu \mathrm{m}$ wide in the CauF — displayed a homogenous distribution of blood capillaries mixed with arterioles, venules, and sporadic lymph capillaries or precollectors. An additional, deep 'Zone C' was a special feature of the CraF (Fig. 4). This zone was poorly vascularised, with only a few blood vascular profiles with larger diameters.

At the crest of CraF and CauF, there was a distinct 'Avascular Area' (blue dashed lines in Fig. 4) located at the transition between Zone A and Zone B.

The common features are graphically displayed in Figure 4. Further findings shall now be described for each zone, and row in greater detail; Figures 5 and 6 illustrate this complex description.

\section{Zone A}

In Zone A, the superficially located Row 1 (Fig. 5) was characterised by capillaries. The arrangement of the capillary profiles within this row was relatively dense. However, the distances between them varied with location; for instance: $37 \mu \mathrm{m}$ (mean value) at the CraF's caudal side vs. $55 \mu \mathrm{m}$ (mean value) at the CraF's crest (Fig. 5). The data indicate that the folds' crests were supplied by capillary networks of relatively low densities (due to wide distances between profiles); this was most distinct in the crest of the CauF. The capillaries' diameters, i.e. the widths of their profiles, were quite uniform (7-9 $\mu \mathrm{m})$ in all locations.

Between the epithelium and the blood capillaries of Row 1, there was always a distinct 'Avascular Band' (Fig. $5^{* *}$, Fig. 6). It was widest at the CraF's and CauF's crests. The width of this Avascular Band was defined as the distances between epithelium and capillary profiles. These distances measured 14-22 $\mu \mathrm{m}$ (Fig. 6), but a few exceptions existed as some blood capillaries were located as close as 5-9 $\mathrm{mm}$ underneath the epithelium.

Row 1 was $37 \mu \mathrm{m}$ (minimum) to $61 \mu \mathrm{m}$ (maximum) wide, depending on the location (Fig. 6).

Row 2 (Fig. 5) contained arterioles and venules. The distances between these vascular profiles were much larger than those in Row 1 (i.e. 132-285 $\mu \mathrm{m}$ ), but - as in Row 1 - they also displayed some topographical differences (Fig. 5). Firstly, in the CauF, the profiles were much closer to each other than in the CraF; in other words, the vascular network of the CauF appeared to be denser than in the CraF. Secondly, the area of the crests of both folds displayed relatively large distances between profiles (indicating 


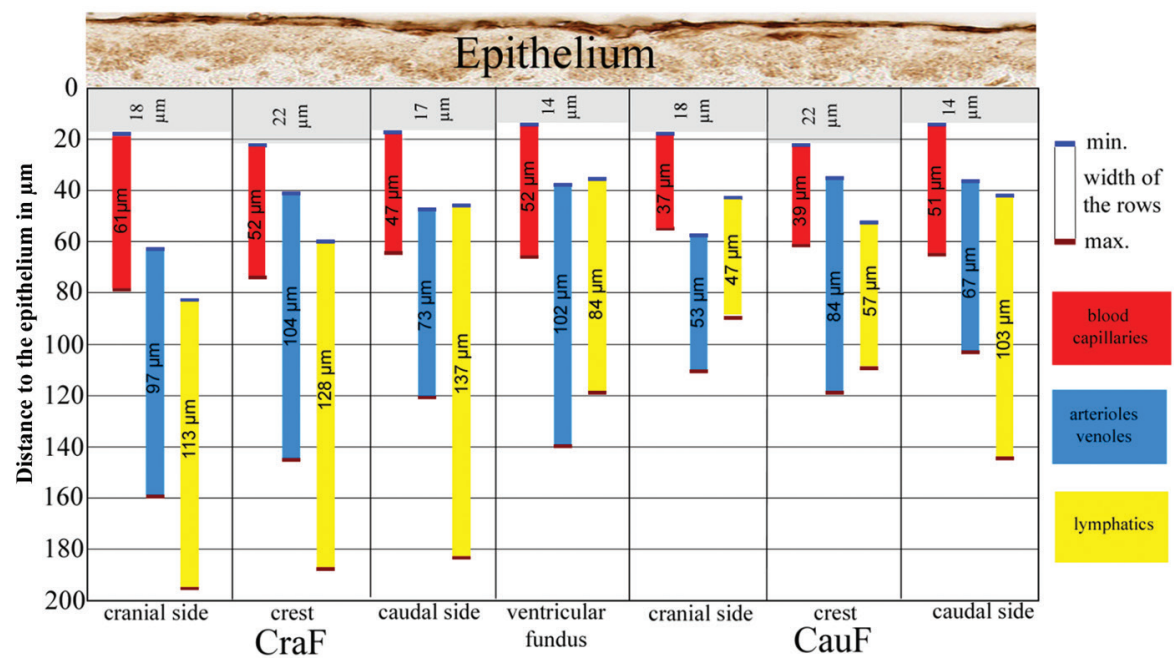

Figure 6. Diagram to display the distances (minimum and maximum) between epithelium and blood capillaries (Row 1), arterioles, venules (Row 2), and lymphatics; the width of these rows (mean values) are written in the coloured columns. The Avascular Band underneath the epithelium (see Fig. $5^{* *}$ ) is indicated in light grey; CraF — cranial fold; CauF — caudal fold.

low vascular density). However, at the cranial sides of both folds, the distances were even larger, and moreover - in the ventricular fundus, the greatest distances of all were encountered (Fig. 5).

The diameters of the arterioles and venules were 16-48 $\mu \mathrm{m}$; the largest were found in the ventricular fundus (Fig. 5). The profiles of the vessels displayed a circular or oval shape; the distribution of both types - circular or oval - was homogenous. These features indicate that in most regions of the cross sections of the vocal folds, the arterioles and venules were cut transversally; only in the ventricular fundus were some of them cut longitudinally.

The vascular profiles of the arterioles and venules - Row 2 - were 35-63 $\mu \mathrm{m}$ away from the epithelium (Fig. 6). Row 2 was much wider than Row 1: Its width was $53 \mu \mathrm{m}$ at a minimum, $104 \mu \mathrm{m}$ at a maximum, depending on the location (Fig. 6).

The Lymphatics, i.e. lymph capillaries and precollectors, were almost exclusively arranged within Row 2. In most regions, the distances between these vascular profiles were greater than those between the arterioles and venules (i.e. 151-254 $\mu \mathrm{m}$ ), although the density of the lymphatics varied with location, too (Fig. 4): In the CauF, the density of the lymphatic profiles was relatively high, particularly at its cranial side.

The diameters of the lymphatics ranged between $13 \mu \mathrm{m}$ and $52 \mu \mathrm{m}$; the largest diameters of the lymphatic profiles were found in the ventricular fundus, i.e. in the same region that contained the largest profiles of the arterioles and venules (Fig. 5).
In most regions of the vocal folds, the lymphatics were cut transversally. Nonetheless, on the CraF's caudal side, and in the ventricular fundus, and on the CauF's cranial side, most of them were cut longitudinally.

Within Row 2, the lymphatic profiles were found in an area which was 47-137 $\mu \mathrm{m}$ wide (Fig. 6). The distances between the lymphatics and the epithelium were quite variable; they ranged from a minimum of 35-83 $\mu \mathrm{m}$ to a maximum of 90-195 $\mu \mathrm{m}$ (Fig. 6).

Special attention should be given to a distinct Avascular Area (brown dashed lines in Fig. 5), which did not contain any microvessels at all. In the CraF, this Avascular Area was larger than in the CauF: 900 $\times 250 \mu \mathrm{m}\left(0.2 \mathrm{~mm}^{2}\right)$ vs. $460 \times 100 \mu \mathrm{m}\left(0.04 \mathrm{~mm}^{2}\right)$; in the $\mathrm{CraF}$, it was located further away from the epithelium when compared to the CauF: $100 \mu \mathrm{m}$ vs. $40 \mu \mathrm{m}$.

\section{Zone B}

Zone B (Fig. 5) was characterised by blood capillaries, arterioles, venules, and lymph capillaries or precollectors. The arrangement of the blood vascular profiles was very homogenous, whereas the lymphatics were found only sporadically. Particularly in the ventricular fundus, lymphatics were detected only in $25 \%$ of the examined minipigs (Fig. 5). Altogether, the vascular network of Zone $B$ was relatively wide when compared with Zone $A$; for instance, the distance between the blood capillaries was $111 \mu \mathrm{m}$ (mean value) in the CraF, and $95 \mu \mathrm{m}$ (mean value) in the CauF (Fig. 5). However, there were some differences 
with respect to the location (Fig. 5): Within the CauF, the profiles of all types of vessels were much closer to each other than in the CraF.

The diameters of the vascular profiles increased towards deeper parts of the lamina propria; only the diameters of the blood capillaries remained constant (Fig. 5). In Zone B, again, it was the area of the ventricular fundus that contained profiles with the largest diameters: 16-91 $\mu \mathrm{m}$ (arterioles, venules) and 57-73 $\mu \mathrm{m}$ (lymphatics). The distribution of the circular and oval profiles in the folds' cross sections was homogenous; most microvessels, however, were cut transversally.

The distance between Zone $B$ and the epithelium was $240 \mu \mathrm{m}$ in the CraF and $270 \mu \mathrm{m}$ in the CauF (measured from the highest point of the crest). The width of Zone B differed markedly: In the CraF, Zone B was quite wide $(1200 \mu \mathrm{m})$, while in the CauF it was only half as wide $(560 \mu \mathrm{m})$.

\section{Zone C}

Zone $\mathrm{C}$ occurred only in the $\mathrm{CraF}$ and was located very deep, i.e. $1440 \mu \mathrm{m}$ away from the epithelium. This was at a proportional level which was, in the CauF, occupied by the thyroarytenoid muscle. Zone $C$ contained homogenously distributed blood capillaries, arterioles and venules. However, no lymphatics were detected. The distances between the vascular profiles were much greater than those of the CraF's and CauF's Zone B. The diameters of the arterioles and venules (21-69 $\mu \mathrm{m}$ ) were much larger, too (Fig. 5).

\section{DISCUSSION}

\section{Animals}

Only female minipigs were included in this examination, because at this point of the study, we wanted to discriminate the sexual dimorphism and the probably related histological differences. The physiological characteristics of the two breeds Göttinger Minipig [37] and Mini-Lewe Minipig [7] appeared to be similar enough not to require a consideration of breed-related differences. The 11-27-month-old animals were in the process of completing skeletal development and adult weight development, and were sexually mature $[42,43]$; accordingly, these animals were classified as adult.

\section{Immunohistochemistry}

The alternating application of the two antibodies - anti-vWF and anti-SMA - facilitated the simulta- neous detection of the blood-vascular and lymphatic-vascular systems in the same tissue sample. Other conventional, established methods such as vascular corrosion casts were not used because they are technically restricted to display only the one or the other vascular system, but not both at the same time. However, as the detection of their topographical relation and of their stratigraphical arrangement in situ (i.e. in the tissue section) was a primary aim of this study, the Corrosion-cast method did not appear appropriate.

\section{Distinction between blood vessels and initial lymphatics: anti-vWF, anti-SMA}

To date, a variety of specific lymphendothelial markers (Prox-1, D2-40, Lyve-1), which are exclusively present on lymphatic endothelial cells, have been proposed to distinguish between blood and lymphatic vessels [40]. These markers: Prox-1 $[22,41$, 48], D2-40 [4, 14, 36], and Lyve-1 [2, 5] have been reported to specifically stain lymphatic endothelial cells in different species. However, in our preliminary studies (unpublished data), none of these commercially available lymphendothelial markers (polyclonal Prox-1, polyclonal Lyve-1, monoclonal D2-40) yielded reliable results in several tested tissues of different organs of the minipig, despite various modifications in the laboratory protocols.

Instead, anti-vWF and anti-SMA were highly efficient for detecting blood and lymph vascular structures and were suitable - by combining of the results of the two procedures - for describing the vascular system adequately.

The vWF is a multimeric glycoprotein of approximately $220 \mathrm{kDa}$ polypeptide units. In the cytoplasm of endothelial cells, vWF is stored in Weibel-Palade bodies $[21,24,29]$. The VWF is a marker of blood vascular and lymphatic endothelium and is therefore regarded as a panendothelial marker. The lymphatic endothelium shows a weaker intensity and more focal staining when compared with blood vascular endothelium because of the sparse and attenuated cytoplasm of the lymphatic endothelial cells $[13,28,30]$.

Smooth muscular alpha-actin is an isoform typical for smooth muscle cells. It is present in high amounts in vascular smooth muscle cells and is also expressed by pericytes. Lymphatic capillaries do not contain smooth muscle cells, and precollectors contain them only sporadically. Therefore, smooth muscle alpha-actin is an appropriate immunohistochemical marker for detecting blood vessels [27, 28, 38, 39], while 
lymphatic capillaries and precollectors are excluded/ /discriminated.

\section{The measurements and the description of the vascular system}

The measurements regarding the microvessels served only as a descriptive tool. A statistical analysis was not intended and did not seem appropriate. For instance, a random test (unpublished data) had shown that in some cases the standard deviations were quite high. Therefore, the measured values were used only as an element of description rather than an element of statistical analysis. As such, the data are valuable to describe the location and topographical relation of the vascular profiles, e.g. in terms of distances from the surface epithelium, or intervascular distances.

\section{The microvascular system as a component of the stratigraphical organisation of the vocal folds}

The measured distances between the epithelium and the vascular profiles were correlated with the topographical subunits of the lamina propria which have been reported by Lang et al. [17], because these proportional subunits comprised 'Regions of Interest (ROI)' of known widths/diameters [17]. Respecting these numerical (metrical) pre-requisites from the literature, each of the characteristic Zones and Rows, as well as the Avascular Band, and the Avascular Area of the microvascular system of the $\mathrm{CraF}$ and the CauF in our study could be allocated to one of these specific layers of the lamina propria: subepithelial, superficial, intermediate, deep layer (SEL, SL, IL, DL; described in the study of Lang et al. [17]): In both vocal folds, the Avascular Band was almost congruent with the SEL, which Lang et al. [17] had characterised by high amounts of fibres per area. Within the underlying SL (a loose region of only few collagen fibres and elastic fibres), the Avascular Area, and Row 1, and Row 2 were located together with the initial lymphatics (all of them components of Zone A). Moreover, in the CraF's crest, the Avascular Area was topographically almost identical with the SL of Lang et al. [17].

Furthermore, in the CraF, the Zone B (with a homogenous distribution of blood capillaries mixed with arterioles, venules, and sporadic initial lymphatics) corresponded to the IL, while Zone C (poorly vascularised) was almost congruent with the DL, which was described by Lang et al. [17] as filled with large amounts of thick collagen fibre bundles.
The situation differed only slightly in the CauF: the first third of Zone B still lay within the SL, the second third within the IL, and the last third was located in the DL. One reason for this was the fact that the CauF lacked Zone $\mathrm{C}$ and deeper parts of the DL. We suggest that the thyroarytenoid muscle of the CauF corresponds functionally and topographically to Zone $C$ and to deeper parts of the DL of the CraF, in accordance with previous descriptions of Lang et al. [17].

\section{Functional considerations with respect to the body-cover model}

According to the body-cover model [11], there is a loose and flexible 'cover' consisting of the epithelium and of the superficial part of the lamina propria. In the region of the cover, the main deformation of the vocal fold occurs [11]. In the minipig, this loose tissue layer was separated from the surface epithelium by a fibro-elastic subepithelial layer, SEL [17]. This SEL was described by Lang et al. [17] as a membrane, which was placed over the loose, soft parts of the vocal folds. In this dense SEL - almost congruent with the Avascular Band - microvessels were markedly sparse. However, underneath - well protected by the densely fibrous SEL - the relatively dense blood-capillary network of Row 1 was located within the SL. Moreover, the Avascular Areas in the region of the folds' crests were a special feature of the $\mathrm{SL}$, which was very loose (with only a few collagen fibres and elastic fibres), especially in the CraF [17]. The functional impact of this finding is discussed below (see: Reinke's space).

Underneath the 'cover', the 'body' comprised the deeper parts of the lamina propria, and - in the porcine CauF (as in the human vocal fold) - the vocalis muscle; it remains rigid during vocal fold vibration $[10,49]$. In the CauF, the deep third of Zone B (adjacent to the vocalis muscle) characterised the body as homogenous, relatively densely vascularised, and supplied with blood capillaries, arterioles, venules, and sporadic initial lymphatics. The same was true for the CraF, but - due to the lack of a muscle - the fibrous part of the body reached further down and comprised the poorly vascularised Zone C.

\section{Reinke's space}

Areas of low fibre density and with large amounts of interstitial amorphous Ground Substance in the human vocal fold had been related with clinical symptoms of Reinke's oedema [15, 32, 33, 35]. These loose tissue areas were referred to as the superficial layer 
of the lamina propria, SLLP, called Reinke's space [12]. In the minipigs' CraF and CauF, there was the above-mentioned, loose, Avascular Area at the level of the superficial layer, SL, which corresponded structurally to Reinke's space in humans. However, care should be taken to respect two distinct features; (1) this Avascular Area had a focal rather than a layered extension, mainly located centrally in the crest of the CraF, and caudally in the crest of the CauF; (2) it was 'covered' by the dense subepithelial layer, SEL [17] which may act as a membrane and therefore have an impact on the spatial limitations of interstitial-fluid expansion towards the mucosal surface. This may be relevant for interpreting something like Reinke's oedema in the pig.

\section{Species-related comparison of the microvascular networks}

The comparison of the microvascular networks of the glottis of pigs and humans displayed both, differences and similarities.

The most striking difference was related to the densities of the blood vascular networks: in humans, the blood-vascular network of the vocal fold has been reported to be denser than that of the vestibular fold [25]. This does not compare with the porcine glottis: Here, the CraF (topographically similar to the human vestibular fold, but presumably the main oscillator in porcine phonation [1]), displayed a vascular network wider than that of the CauF (topographically identical to the human vocal fold).

The most striking similarities existed in terms of the stratigraphical organisation of networks, and course (direction) of the vessels. In the human glottis, blood capillaries form a superficial network, while blood vessels with larger calibres are located below [23, 25, 34]; lymphatic microvessels form a superficial, close-meshed network, and a deep, wide-meshed network [16, 18-20,44-47, 34]. This conforms to the situation in the porcine glottis.

The shapes of the blood microvascular profiles in the porcine specimens suggested that the vascular orientation was mostly parallel to the long axes of the folds, entering the latter from the dorsal and ventral commissures; this has been described accordingly in the human glottis $[23,25,34]$. In terms of function, authors have regarded the blood vascular organisation of the human glottis as being well suited to prevent disturbances of the circulation and metabolism of the tissues; such disturbances might be caused by vibration during phonation $[23,25]$. With regard to the above mentioned structural similarities, the same can be assumed also for the porcine glottis.

\section{CONCLUSIONS}

The orientation of the lymphatic vessels was quite similar to that of the blood vessels: In humans, the lymphatics of the free edge of the vocal fold run in the direction of the fold's long axis, while the lymphatics in the ventricle and in the area of the subglottis run perpendicular to the vocal fold's long axis [18, 34, 44, 45]. In the porcine glottis, the situation is basically the same, but two peculiar findings made the system more special: many lymphatic vessels on the CraF's caudal side, and in the ventricular fundus, and on the CauF's cranial side ran perpendicular to the folds' long axes. The direction of lymph drainage - towards the arytenoid region and towards the ventricular fundus in the CraF and CauF - appears to be analogous to that in the human vocal fold $[18,34,44,45]$.

\section{REFERENCES}

1. Alipour F, Jaiswal S (2008) Phonatory characteristics of excised pig, sheep, and cow larynges. J Acoust Soc Am, 123: 4572-4581.

2. Banerji S, Ni J, Wang SX, Claper S, Su J, Tammi R, Jones M, Jackson DG (1999) LYVE-1, a new homologue of the CD44 glycoprotein, is a lymph-specific receptor for hyaluronan. J Cell Biol, 144: 789-801.

3. Chan RW, Fu M, Young L, Tirunagari N (2007) Relative contributions of collagen and elastin to elasticity of the vocal fold under tension. Ann Biomed Eng, 35: 1471-1483.

4. Evangelou E, Kyzas PA, Trikalinos TA (2005) Comparison of the diagnostic accuracy of lymphatic endothelium markers: Bayesian approach. Mod Pathol, 18: 1490-1497.

5. Furukawa M, Shimoda H, Kajiwara T, Kato S, Yanagisawa S (2008) Topographic study on nerve-associated lymphatic vessels in the murine craniofacial region by immunohistochemistry and electron microscopy. Biomed Res, 29: 289-296.

6. Gray SD (2000) Cellular physiology of the vocal folds. Otolaryngol Clin North Am 33: 679-698.

7. Gundlach M (2012) Phänotypische Charakterisierung des Wachstums ausgewählter Röhrenknochen an Vorderund Hinterextremität bei Miniaturschweinen der Rasse 'Mini-LEWE' mittels quantitativer Computertomographie (doctoral dissertation). Stiftung Tierärztliche Hochschule Hannover, Hannover.

8. Hahn MS, Jao CY, Faquin W Grande-Allen KJ (2008) Glycosaminoglycan composition of the vocal fold lamina propria in relation to function. Ann Otol Rhinol Laryngol, 117: 371-381.

9. Haji T, Mori K, Omori K, Isshiki N (1992) Experimental studies on the viscoelasticity of the vocal fold. Acta Otolaryngol, 112: 151-159.

10. Hirano M (1974) Morphological structure of the vocal cord as a vibrator and its variations. Folia Phoniatr, 26: 89-94. 
11. Hirano M (1981) Clinical examination of voice. Springer, Wien, New York.

12. Hirano M, Kurita S, Nakashima T (1983) Growth, development, and aging of human vocal cords. In: Bless DM, Abbs JH eds. Vocal fold physiology, contemporary research and clinical issues. Cottage-Hill Press, San Diego, pp. 22-43.

13. Hultberg BM, Svanholm H (1989) Immunohistochemical differentiation between lymphangiographically verified lymphatic vessels and blood vessels. Virchows Arch A Pathol Anat Histopathol, 414: 209-215.

14. Kaiserling E (2004) Immunhistochemische Darstellung von Lymphgefäßen mit D2-40 in der diagnostischen Patholgie. Pathologe, 25: 362-374.

15. Kambic V, Gale N, Radsel Z (1989) Die anatomischen Merkmale des Reinke-Raumes und die Ätio-Pathogenese des Reinke-Ödems. Laryngo-Rhino-Otol, 68: 231-235.

16. Kubik S (2002) Anatomie des Lymphgefäßsystems. In: Földi $M$, Kubik S eds. Lehrbuch der Lympholgie. $5^{\text {th }}$ Ed. Urban and Fischer, München, Jena, pp. 1-179.

17. Lang A, Koch R, Rohn K, Gasse H. Histomorphometric analysis of collagen and elastic fibres in the cranial and caudal fold of the porcine glottis. Anat Histol Embryol, doi: 10.1111/ahe.12125 [In press].

18. Liu YH, Xu SC, Tu LL, Zhang KL, Lu DH, Zhang M (2006) A rich lymphatic network exists in the inferior surface of the vocal cord. Surg Radiol Anat, 28: 125-128.

19. Mann W (1979) The lymphatic system of the larynx. A lyphangioscopic and ultrastructural study (author's transl.). Eur Arch Oto-Rhino-Laryngol, 225: 165-180.

20. Mann W, Beck Chl (1979) On the ultrastructure of laryngeal lymphatics. Eur Arch Oto-Rhinso-Laryngol, 223: 278-281.

21. Mannucci PM (1998) von Willebrand factor: a marker of endothelial damage? Arterioscler Thromb Vasc Biol, 18: 1359-1362.

22. Martin A, Gasse H, Staszyk C (2010) Absence of lymphatic vessels in the dog dental pulp: an immunohistochemical study. J Anat, 217: 609-615.

23. Mihashi S, Okada M, Kurita S, Nagata K, Oda M, Hirano M, Nakashima T (1981) Vascular network of the vocal fold. In: Stevens KN, Hirano M ed. Vocal cord physiology. University of Tokyo, Tokyo, pp. 45-47.

24. Müller AM, Hermanns MI, Skrzynski C, Nesslinger M, Müller K-M, Kirkpatrick CJ (2002) Expression of the Endothelial markers PECAM-1, VWF, and CD34 in vivo and in vitro. Experimental Molecular Patholgie, 72: 221-229.

25. Nakai Y, Masutani H, Moriguchi M, Matsunaga K, Sugita M (1991) Microvascular structure of the larynx. A scanning electron microscopic study of microcorrosion casts. Acta Otolaryngol Suppl, 486: 254-263.

26. Nita LM, Battlehner CN, Ferreira MA, Imamura R, Sennes LU, Caldini EG, Tsuji DH (2009) The presence of a vocal ligament in fetuses: a histochemical and ultrastructural study. J Anat, 215: 692-697.

27. Nehls V, Drenckhahn D (1991) Heterogeneity of microvascular pericytes for smooth muscle type alpha-actin. J Cell Biol, 113: 147-154.

28. Paavonen K, Puolakkainen P, Jussila L, Jahkola T, Alitalo K (2000) Vascular endothelial growth factor receptor-3 in lymphangiogenesis in wound healing. Am J Pathol, 156: 1499-1504.

29. Poblet E, Gonzalez-Palacios F, Jimenez FJ (1996) Different immunoreactivity of endothelial markers in well and poorly differentiated areas of angiosarcomas. Virchows Arch, 428: 217-221.

30. Pusztaszeri MP, Seelentag W, Bosman FT (2006) Immunohistochemical expression of endothelial markers CD31, CD34, von Willebrand factor, and Fli-1 in normal human tissues. Histochem Cytochem, 54: 385-395.

31. Reinhard H, Koch R, Gasse H (2013) Histomorphometric analysis of the lymphatic and blood vascular system of the glottis of adult pigs. Poster 15: www.anatomischegesellschaft.de/Tagungen-ag3/abstract-archive.html, S. 74; accessed January 27, 2014.

32. Reinke F (1897) Über die funktionelle Struktur der menschlichen Stimmlippe mit besonderer Berücksichtigung des elastischen Gewebes. Anatomische Hefte, 9: 103-116.

33. Remenár E, Élö J, Frint T (1984) The morphological basis for development of Reinke's oedema. Acta Oto-Laryngol, 97: 169-176.

34. Roberts T, Morton R, Al-Ali S (2011) Microstructure of the vocal fold in elderly humans. Clin Anat, 24: 544-551.

35. Sakae FA, Imamura R, Sennes LU, Mauad T, Saldiva PH, Tsuji DH (2008) Disarrangement of collagen fibers in Reinke's edema. Laryngoscope, 118: 1500-1503.

36. Saito Y, Nakagami H, Morishita R, Takami Y, Kikuchi Y, Hayashi H, Nishikawa T, Tamai K, Azuma N, Sasajima T, Kaneda Y (2006) Transfection of human hepatocyte growth factor gene ameliorates secondary lymphedema via promotion of lymphangiogenesis. Circulation, 114: 1177-1184.

37. Simianer H, Köhn F (2010) Genetic management of the Göttingen Minipig population. J Pharmacol Toxicol Methods, 62: 221-226.

38. Skalli O, Ropraz P, Trzeciak A, Benzonana G, Gillessen D, Gabbiani G (1986) A monoclonal antibody against alpha-smooth muscle actin: a new probe for smooth muscle differentiation. J Cell Biol, 103: 2787-2796.

39. Skalli O, Pelte MF, Peclet MC, Gabbiani G, Gugliotta P, Bussolati G, Ravazzola M, Orci L (1989) Alpha-smooth muscle actin, a differentiation marker of smooth muscle cells, is present in microfilamentous bundles of pericytes. Histochem Cytochem, 37: 315-321.

40. Sleeman JP, Krishnan J, Kirkin V, Baumann P (2001) Markers for the lymphatic endothelium: in search of the holy grail? Microsc Res Tech, 55: 61-69.

41. Staszyk C, Duesterdieck KF, Gasse H, Bienert A (2005) Immunohistochemical identification of lymphatic vessels in the peridontium of equine cheek teeth. J Vet Dent, 22: 227-232.

42. Swindle MM, Smit AC, Laber K, Goodrich JA, Bingel SA (2006) Biology and medicine of swine. In: Reuter JD, Suckow MA eds. Laboratory Animal Medicine and Management, International Veterinary Information Service. Ithaca, New York.

43. Swindle MM, Makin A, Herron AJ, Clubb FJ Jr, Frazier KS (2012) Swine as Models in Biomedical Research and Toxicology Testing. Vet Pathol, 49: 344-356.

44. Werner JA, Schünke M, Rudert H, Tillmann B (1990) Description and clinical importance of the lymphatics and vocal fold. Otolaryngol Head Neck Surg, 102: 13-19.

45. Werner JA, Schünke M, Rudert H (1992) Histochemische, injektionstechnische, licht- und transmissionselektronenmikroskopische Untersuchungen zum laryngealen Lymphgefäßsystem des Menschen. In: Behrens v. Rautenfeld, Ausgewählte Vorträge der Lymphologica' 91 Hannover, Kagerer-Kommunikation, Bonn.

46. Werner JA, Schünke M, Lippert BM, Koeleman- Schmidt H, Gottschlich S, Tillmann B (1995) Das laryngeale Lymphgefäßsystem des Menschen. HNO, 43: 525-531.

47. Werner JA, Dunne AA, Myers JN (2003) Functional anatomy of the lymphatic drainage system of the upper aerodigestive tract and its role in metastasis of squamous cell carcinoma. Head Neck, 25: 322-332.

48. Wilting J, Papoutsi M, Christ B, Nicolaides KH, von Kaisenberg CS, Stark J, Alitalo K, Tomarev SI, Niemeyer C, Rossler J (2002) The transcription factor Prox 1 is a marker for lymphatic endothelial cells in normal and diseased human tissues. FASEB J, 16: 1271-1273.

49. Yumoto E, Kadota Y (1998) Pliability of the vocal fold mucosa in relation to the mucosal upheaval during phonation. Arch Otolaryngol Head Neck Surg, 124: 897-902. 